\title{
A 4-year study of the efficacy and tolerability of enzyme replacement therapy with agalsidase alfa in 36 women with Fabry disease
}

\author{
Catharina Whybra, $M D^{I}$, Elke Miebach, $M D^{I}$, Eugen Mengel, $M D^{I}$, Andreas Gal, $M D^{2}$, \\ Karin Baron, $M D^{I}$, Michael Beck, $M D^{1}$, and Christoph Kampmann, $M D^{I}$
}

\begin{abstract}
Purpose: Although Fabry disease is $\mathrm{X}$ linked and considered to affect primarily male hemizygotes, female heterozygotes may experience all the signs and symptoms of this metabolic disorder. This prospective, singlecenter, open-label, clinical trial was performed to evaluate the long-term response of female patients with Fabry disease to enzyme replacement therapy. Methods: Symptomatic women (average age $=47$ years) enrolled in this 4-year study were treated with agalsidase alfa (Replagal ${ }^{\circledR}$, Shire HGT, Inc.) at a dose of $0.2 \mathrm{mg} / \mathrm{kg}$, every other week for 4 years $(N=36)$. Clinical and biochemical assessments were conducted at 12-month intervals. Results: The Mainz Severity Score Index, a measure of total disease burden, was significantly reduced after 12 months $(P<0.01)$ of treatment and continuously improved over 4 years. Brief Pain Inventory "pain at its worst" score was reduced from $4.6 \pm 2.9$ at baseline to $3.3 \pm 2.9$ after 12 months $(P=0.001)$ and remained reduced through 4 years. Mean leftventricular mass decreased from $89.4 \pm 29.3 \mathrm{~g} / \mathrm{m}^{2.7}$ at baseline to $66.5 \pm$ $29.3 \mathrm{~g} / \mathrm{m}^{2.7}$ after 12 months $(P<0.001)$ and remained reduced through 4 years. Average kidney function (estimated glomerular filtration rate and proteinuria) remained constant during the study. No safety issues were identified. Conclusions: Long-term agalsidase alfa is effective and was well tolerated in women with Fabry disease. Genet Med 2009:11(6): 441-449.
\end{abstract}

Key Words: Fabry disease, agalsidase alfa, females, lysosomal storage disease, cardiomyopathy

$\mathrm{F}_{\mathrm{t}=\mathrm{a}}$ bry disease is an X-linked error of glycosphingolipid metabolism caused by a deficiency in the activity of the lysosomal enzyme, $\alpha$-galactosidase A (GALA). ${ }^{1}$ In affected individuals, the enzyme substrate, globotriaosylceramide (Gb3), accumulates in cells and organs, where it participates by a yet unknown mechanism in the pathologies that are characteristic of Fabry disease. ${ }^{1}$ Disease manifestations include acroparasthesias and neuropathic pain crises $^{2-4}$; angiokeratomas; cardiac involvement, including left-ventricular hypertrophy (LVH),

From the ${ }^{1}$ Center for Lysosomal Storage Diseases, Children's Hospital of the Johannes Gutenberg University, Mainz, Germany; and ${ }^{2}$ Institute for Human Genetics, University Hospital Eppendorf, Hamburg, Germany.

Dr. Christroph Kampmann, Universitätskinderklinik, Johannes Gutenberg Universität, Langenbeckstr. 1, D-55101 Mainz, Germany.E-mail: kampmann@ mail.uni-mainz.de.

Disclosure: M. Beck reports receiving honoraria, travel grants, or research grants from Shire Human Genetic Therapies, Inc., and Genzyme, Inc, and Biomarin, Inc. E. Miebach, A. Gal, and E. Mengel report receiving honoraria, travel grants, or research grants from Shire Human Genetic Therapies, Inc. and Genzyme, Inc. The remaining authors report receiving honoraria, travel grants, or research grants from Shire Human Genetic Therapies, Inc.

Submitted for publication November 25, 2008

Accepted for publication February 23, 2009.

Published online ahead of print April 2, 2009

DOI: $10.1097 /$ GIM.0b013e3181a23bec valvular changes, ischemia, and myocardial infarction 5 ; cerebrovascular abnormalities resulting in stroke ${ }^{6,7}$; autonomic nervous system dysfunction causing decreased sweat function $^{8}$ and reduced heart-rate variability ${ }^{9,10}$; and kidney dysfunction progressing to end-stage renal disease (ESRD). ${ }^{11}$ Fabry disease occurs in people of all ethnicities with an estimated incidence of about 1 in 117,000 male births, ${ }^{12}$ although newborn screening studies have suggested a much higher incidence. ${ }^{13}$

Heterozygotes with Fabry disease are commonly designated as "carriers" that by definition indicates the presence of a mutated allele. In X-linked conditions, the term "carrier" does not define the phenotype (i.e., the clinical expression of disease), which is often a source of misunderstanding in counseling or communications. Like in some X-linked conditions, ${ }^{14}$ heterozygous women may be symptomatic ${ }^{15,16}$ but with an onset and rate of progression that is more variable than that observed in men. ${ }^{15-22}$ The onset of signs and symptoms of Fabry disease occurs during childhood and adolescence in girls, just as it does in boys. ${ }^{4,23,24}$ However, the onset of major organ involvement (kidney, heart, brain) occurs about $6-10$ years later in women than in men. ${ }^{15,19,25-30}$ The burden of disease in women can be substantial. For example, the Mainz Severity Score Index (MSSI) shows that men and women experience a similar impact from Fabry disease, ${ }^{31}$ and health-related quality of life is similarly reduced in women and men with Fabry disease. 15,18,32-34 $^{2}$ Without treatment, lifespan is typically reduced by 15 years in women with Fabry disease. ${ }^{19}$

Agalsidase alfa (Replagal ${ }^{\circledR}$, Shire Human Genetic Therapies, Inc., Cambridge, MA), a human form of GALA manufactured in a human cell line by gene activation, ${ }^{35}$ has been tested in men, women, and children. In men treated with agalsidase alfa, the severity of neuropathic pain was reduced, ${ }^{36}$ kidney function was stabilized in patients with Stage 1 or Stage 2 chronic kidney disease (CKD) at baseline, ${ }^{37}$ and left-ventricular mass (LVM) was reduced in patients with LVH at baseline. ${ }^{38}$ In children with neuropathic pain, 6 months of agalsidase alfa treatment resulted in a significant reduction or cessation of the use of neuropathic pain medications, and in boys, 6 months of agalsidase alfa treatment significantly improved abnormal heart-rate variability. ${ }^{9}$ A single open-label study of agalsidase alfa in 15 women has been reported, and it showed a significant reduction in LVM and statistically significant improvement in quality of life. ${ }^{39}$ Here, we present the results of a 4-year study of agalsidase alfa in a cohort of 36 women with Fabry disease.

\section{MATERIALS AND METHODS}

\section{Study design}

Women aged 18 years and older with a confirmed diagnosis of Fabry disease were eligible for enrollment in this prospective, 
single-center, open-label study provided that they were experiencing manifestations of Fabry disease and had not been previously treated with enzyme replacement. Mutation analysis was performed to confirm the diagnosis of Fabry disease. The protocol was approved by the ethics committee of the Mainz University Hospital, and all patients signed informed, written consent before enrollment.

During the period from January 2001 to July 2004, a total of 86 female patients with a confirmed diagnosis of Fabry disease were examined at the Mainz center. Of these patients, 15 were ineligible for inclusion because they were younger than 18 years, and 31 were not offered enzyme replacement therapy (ERT) because they were not experiencing any debilitating symptoms (e.g., neuropathic pain, gastrointestinal involvement, stroke) or classic signs of major organ involvement (e.g., proteinuria, decreased glomerular filtration rate, $\mathrm{LVH}$ ). Thus, the study population consisted of 40 women with signs and symptoms of Fabry disease who were treated with agalsidase alfa. Fifteen of these 40 patients had participated in the previously reported Shire HGT-sponsored clinical trial of agalsidase alfa in women $^{39}$ for 13-41 weeks, and their extension results are presented here.

\section{Treatment}

All patients were treated with agalsidase alfa at a dose of 0.2 $\mathrm{mg} / \mathrm{kg}$ infused intravenously every other week. The infusion took place over 40 minutes and was given without premedication. After completion of the clinical study noted above, ${ }^{39}$ all patients were treated with commercially obtained drug.

\section{Measurements}

Patients were assessed at baseline and at 12-month intervals. The following measurements were performed: estimated glomerular filtration rate (eGFR) and proteinuria; plasma Gb3 and urine Gb3; LVM and New York Heart Association (NYHA) functional score, Brief Pain Inventory (BPI), ${ }^{40}$ and MSSI. ${ }^{31}$ When using the MSSI, a value is assigned to prevalent signs and symptoms in four areas of involvement (neurologic, renal, cardiovascular, general). ${ }^{31}$ A higher total score indicates a larger total burden of the disease. Thus, an improvement during treatment with agalsidase alfa would be seen as a decrease in MSSI. eGFR was calculated using the abbreviated Modification of Diet in Renal Disease equation, ${ }^{41}$ and proteinuria was based on a 24-hour urine collection. The change in eGFR during treatment was analyzed in subgroups according to the classification of CKD. ${ }^{42}$ A responder analysis for kidney function was performed comparing the final assessment to the baseline assessment using two different definitions of a responder: (1) $<20 \%$ decrease in GFR and (2) no shift to a more severe state of CKD. ${ }^{43}$ LVM was assessed by standard echocardiographic techniques and was performed by a single, experienced, boardcertified cardiologist. LVM was calculated according to the equations of Devereux ${ }^{44}$ and was indexed to height to the 2.7 power (LVMi in $\mathrm{g} / \mathrm{m}^{2.7}$ ). LVH was defined as having an LVM $>48 \mathrm{~g} / \mathrm{m}^{2.7} .45$ During the conduct of this study, plasma and urine Gb3 levels were determined at two different clinical laboratories using methods similar to those previously described in detail. ${ }^{35}$ Because the laboratories reported different limits of normal values and used different units of measurement (the reference values in the non-Fabry population at these laboratories were: plasma $\mathrm{Gb} 3<3.3 \mu \mathrm{mol} / \mathrm{L}$ and $<4 \mu \mathrm{g} / \mathrm{mL}$; urine $\mathrm{Gb} 3$ $<0.6$ molar ratio of Gb3 to sphingomyelin and $<0.03 \mathrm{mg} / \mathrm{mmol}$ Gb3/creatinine), the values were converted to a 3-point scale as follows: (1) in the normal range; (2) $>$ normal, but $\leq 2$ times the upper limit of normal (ULN); and (3) $>2$ times the ULN.
Activity of GALA in peripheral leukocytes and concentrations of Gb3 in urine and plasma were determined by methods previously described. ${ }^{35}$

\section{Safety}

Safety was assessed continuously during the study by monitoring adverse events. In addition, the presence of antiagalsidase alfa antibodies was determined using a validated enzymelinked immunosorbent assay. ${ }^{36}$

\section{Statistics}

Methods of descriptive statistics were used. Linear regression was used to evaluate the relationship between age (or age squared in the case of $\mathrm{LVM}^{26}$ ) and baseline measures of eGFR, LVM, and MSSI. Repeated measures ANOVA were used to evaluate the effect of agalsidase alfa on eGFR, LVM, MSSI, NYHA heart failure classification, and log-transformed proteinuria. Proteinuria data from three patients who were being treated with corticosteroids for nephropathies were excluded from the analysis. Linear contrasts were used to compare mean values at each time point to baseline values. All calculations were done using JMP version 3.2.2 (SAS Institute, Cary, NC). A $P$ value $<0.05$ was considered to be statistically significant.

Treatment was interrupted for four women during this study (after $0.7,0.8,1.8$, and 2.2 years) due to termination of medical insurance. Data from these patients were included in the baseline cross-sectional analyses but, because of their short period of treatment, were not included in the longitudinal analyses. For the remaining patients, last observation carried forward was used as an estimate of missing values.

\section{RESULTS}

\section{Demographics and cross-sectional assessment of disease manifestation in women}

A total of 40 women with Fabry disease enrolled in this study. With the exception of one 14-year-old adolescent, all were at least 18 years old. Their average age was $47.0 \pm 17.9$ (mean $\pm \mathrm{SD}$ ) with a range from 14 to 76 years. The mutations and leukocyte GALA activities are presented in Table 1. Ten women had GALA activities below the lower limit of normal (normal range: $0.23-1.14 \mathrm{mU} / \mathrm{mg}^{39}$ ). All women were symptomatic at baseline with an average MSSI of $26.2 \pm 12.3$ (mean $\pm \mathrm{SD}$ ). Thirty-six $(90 \%)$ of the patients completed 4 years of treatment without interruption. The remaining four women completed $0.7,0.8,1.8$, and 2.2 years before an interruption in treatment. The duration of the treatment interruption in these four women was $2.5,2.5$, permanent, and 1.2 years, respectively.

Figure 1 presents a cross-sectional analysis of the association between age (or age squared in the case of LVMi) at baseline and MSSI, eGFR, LVMi, and BPI "pain at its worst" score. At baseline, individual MSSI scores were significantly correlated with age (Fig. 1), indicating an increasing total burden of Fabry disease with increasing age. The average eGFR at baseline was $91.0 \pm 29.7$, and eGFR significantly declined with age at a rate of about $1.16 \mathrm{~mL} /$ minute $/ 1.73 \mathrm{~m}^{2}$ per year. In contrast, neither proteinuria nor log-transformed proteinuria was found to change with age (slope of the proteinuria versus age line was $-7.9 \mathrm{mg} /$ day per year, $P=0.55)$, and the slope of the logtransformed proteinuria versus age line was -0.00037 (log $\mathrm{mg}$ )/day per year; $P=0.94$ (data not shown). The severity of 
Table 1 GALA mutations and enzyme activity in peripheral leukocytes

\begin{tabular}{|c|c|c|c|c|c|}
\hline $\mathrm{Pt}$ & Age (yr) & Mutation & Exon & $\begin{array}{l}\text { GALA activity } \\
(\mathrm{mU} / \mathrm{mg})^{a}\end{array}$ & Mutation type \\
\hline 30 & 29 & c. $34 \mathrm{del} 24$ & 1 & 0.361 & $\begin{array}{l}\text { Deletion (results in deletion of eight amino acids } \\
\text { in the signal peptide required for targeting) }\end{array}$ \\
\hline 31 & 31 & c.34del24 & 1 & 0.8771 & $\begin{array}{l}\text { Deletion (results in deletion of eight amino acids } \\
\text { in the signal peptide required for targeting) }\end{array}$ \\
\hline 12 & 54 & p.C52S & 1 & 0.7657 & Missense \\
\hline 28 & 25 & p.H46R & 1 & 0.3548 & Missense \\
\hline 29 & 48 & p.H46R & 1 & 1.0202 & Missense \\
\hline 39 & 45 & c. $270 \mathrm{delC}$ & 2 & 0.3 & Single nucleotide deletion (would result in a frameshift) \\
\hline 9 & 50 & $\mathrm{IVS} 2+1 \mathrm{G}>\mathrm{A}$ & 2 & 0.1567 & $\begin{array}{l}\text { Single nucleotide change at the beginning of intron } \\
2 \text {, affecting correct splicing of the GALA mRNA }\end{array}$ \\
\hline 26 & 36 & $\mathrm{IVS} 2+1 \mathrm{G}>\mathrm{A}$ & 2 & 1.008 & $\begin{array}{l}\text { Single nucleotide change at the beginning of intron } \\
2 \text {, affecting correct splicing of the GALA mRNA }\end{array}$ \\
\hline 34 & 41 & $\mathrm{IVS} 2+1 \mathrm{G}>\mathrm{A}$ & 2 & 0.5853 & $\begin{array}{l}\text { Single nucleotide change at the beginning of intron } \\
2 \text {, affecting correct splicing of the GALA mRNA }\end{array}$ \\
\hline 11 & 25 & p.R112H & 2 & 0.322 & Missense \\
\hline 2 & 39 & p.G147R & 3 & 0.33 & Missense \\
\hline 16 & 19 & p.G147R & 3 & 0.1 & Missense \\
\hline 23 & 41 & p.L129P & 3 & 0.4597 & Missense \\
\hline 6 & 66 & p.L131P & 3 & 0.6629 & Missense \\
\hline 25 & 60 & c.718delAA & 5 & 0.5414 & Frameshift (deletion of two nucleotides) \\
\hline 27 & 37 & c.718delAA & 5 & 0.7249 & Frameshift (deletion of two nucleotides) \\
\hline 17 & 73 & p.R220X & 5 & 0.0637 & Nonsense \\
\hline 18 & 72 & p.R220X & 5 & 0.1046 & Nonsense \\
\hline 19 & 76 & p.R220X & 5 & 0.2081 & Nonsense \\
\hline 20 & 74 & p.R220X & 5 & 0.1672 & Nonsense \\
\hline 36 & 57 & p.R220X & 5 & 1.237 & Nonsense \\
\hline 40 & 71 & p.R220X & 5 & 0.7955 & Nonsense \\
\hline 7 & 58 & p.R227Q & 5 & 0.8231 & Missense \\
\hline 4 & 72 & p.W236C & 5 & 0.83 & Missense \\
\hline 5 & 42 & p.W236C & 5 & 0.4995 & Missense \\
\hline 3 & 65 & c. $912 \mathrm{delC}$ & 6 & 0.14 & Frameshift (single nucleotide deletion) \\
\hline 8 & 43 & p.A288D & 6 & 0.409 & Missense \\
\hline 10 & 46 & p.N320I & 6 & 0.4964 & Missense \\
\hline 22 & 39 & p.N320I & 6 & 0.19 & Missense \\
\hline 15 & 33 & p.R301X & 6 & 0.48 & Missense \\
\hline 37 & 26 & p.Q321X & 6 & 0.1764 & Nonsense \\
\hline 1 & 35 & p.R301X & 6 & 1.0897 & Nonsense \\
\hline 21 & 59 & p.R301X & 6 & 0.591 & Nonsense \\
\hline 33 & 21 & p.R301X & 6 & 1.031 & Nonsense \\
\hline 38 & 22 & c.1072delGAG & 7 & 0.04 & In-frame deletion (three nucleotides) \\
\hline
\end{tabular}




\begin{tabular}{lclccc}
\hline \multicolumn{1}{l}{ Table 1 Continued } & & & \\
\hline $\mathrm{Pt}$ & Age (yr) & Mutation & Exon & GALA activity (mU/mg) $^{a}$ & Mutation type \\
\hline 13 & 67 & p.A350P & 7 & 0.4981 & Missense \\
14 & 43 & p.A350P & 7 & 0.7324 & Missense \\
35 & 14 & p.A350P & 7 & 0.3938 & Missense \\
24 & 52 & p.W340X & 7 & 0.703 & Nonsense \\
32 & 75 & p.W340X & 7 & 1.2971 & Nonsense \\
\hline
\end{tabular}

The values below the lower limit of normal are indicated in bold.

${ }^{a}$ The range in nonaffected women is $0.23-1.14 \mathrm{mU} / \mathrm{mg}$.

neuropathic pain, as measured by the BPI "pain at its worst" score, was not related to baseline age.

\section{Mainz Severity Score Index}

As shown in Figure 2, MSSI was significantly decreased after the first year of treatment and remained significantly reduced through 4 years. Changes in the neurologic and cardiovascular subscores were responsible for most of the change in MSSI during treatment with agalsidase alfa (Fig. 3). Of the six patients in the "severe" range at baseline (MSSI $\geq 40^{31}$ ), four exhibited decreases into the "moderate" range (MSSI $\geq 20$ and $<40$ ). Similarly, 8 of 18 patients in the moderate range at baseline had MSSI scores in the "mild" range $($ MSSI $<20)$ after 4 years of treatment. A single patient, who was in the mild range at baseline, demonstrated a shift into the next higher range after 4 years of treatment.

\section{Kidney function}

In the 36 patients who completed 4 years of treatment, the overall mean eGFR was $91.0 \pm 31.2 \mathrm{~mL} / \mathrm{minute} / 1.73 \mathrm{~m}^{2}$ at baseline and $91.0 \pm 25.6 \mathrm{~mL} /$ minute $/ 1.73 \mathrm{~m}^{2}$ after 4 years of agalsidase alfa (Table 2). The four patients with eGFR $>135$ $\mathrm{mL} /$ minute $/ 1.73 \mathrm{~m}^{2}$ at baseline (defined as hyperfiltration ${ }^{46}$ ) experienced a reduction of eGFR during the study, with average eGFR declining from $159.0 \pm 17.9 \mathrm{~mL} /$ minute $/ 1.73 \mathrm{~m}^{2}$ at baseline to $128.3 \pm 4.6 \mathrm{~mL} / \mathrm{minute} / 1.73 \mathrm{~m}^{2}$ after 4 years (range at 4 years, $125-135 \mathrm{~mL} /$ minute $/ 1.73 \mathrm{~m}^{2}$ ). Patients with Stage 1 and Stage 3 CKD remained essentially stable during 48 months of treatment, with only one of nine patients with Stage $1 \mathrm{CKD}$ progressing to Stage $2 \mathrm{CKD}$ and with none of the three patients with Stage 3 CKD progressing to Stage 4 CKD. The subgroup of 20 patients with moderately reduced kidney function at
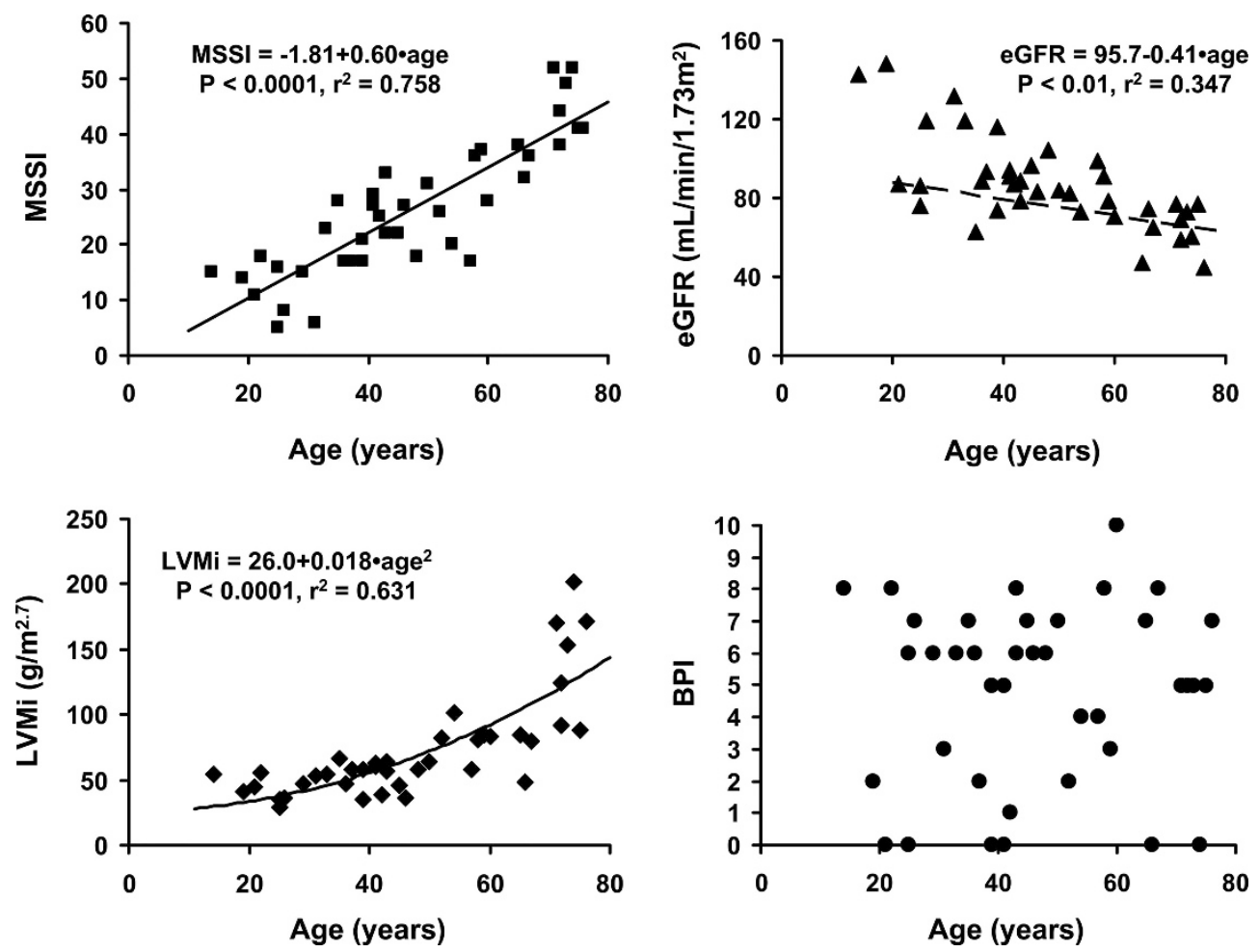

Fig. 1. The association of baseline characteristics of Fabry disease with age in women with Fabry disease. The dashed line in the eGFR plot is the regression analysis for the 25 patients with baseline eGFR $<90 \mathrm{~mL} / \mathrm{minute} / 1.73 \mathrm{~m}^{2}$. No significant association between age and baseline Brief Pain Index (BPI) "pain at its worst" score was found. 


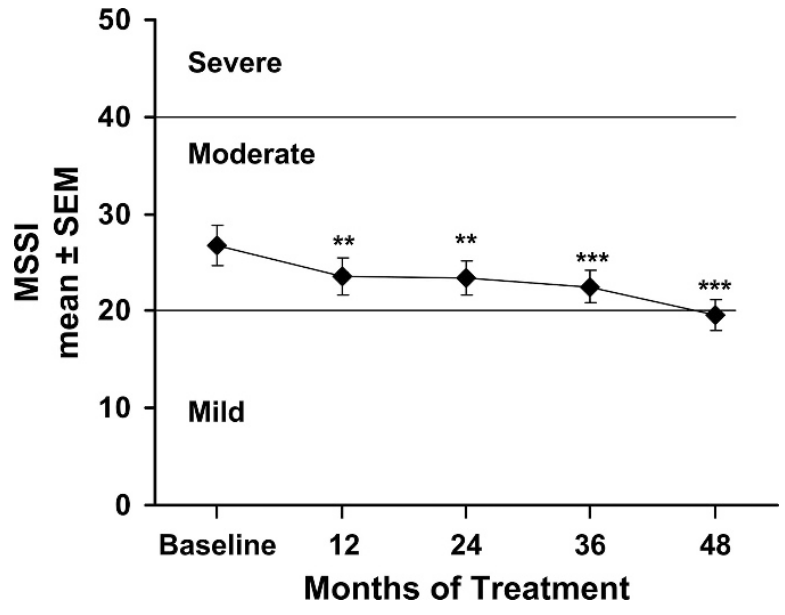

Fig. 2. The effect of agalsidase alfa on MSSI in women with Fabry disease. ${ }^{*} P<0.05,{ }^{* *} P<0.01,{ }^{* * *} p<0.001$ compared with baseline value. $N=36$. The horizontal lines indicate the boundaries for MSSI scores associated with mild, moderate, and severe involvement. ${ }^{31}$

baseline (Stage $2 \mathrm{CKD}$, eGFR $>60$ and $\leq 90 \mathrm{~mL} /$ minute $/ 1.73$ $\mathrm{m}^{2}$ ) demonstrated a significant increase in eGFR after 1 year of treatment, and eGFR remained improved through 4 years (Table 2). One patient in this subgroup had declined to CKD Stage 3 after 3 years of treatment, and another patient had become a hyperfiltrator after 4 years. Responder analysis for the women who completed 4 years of ERT and who were not hyperfiltrating at baseline $(n=32)$ is shown in Table 3. By these criteria, $>90 \%$ of the women demonstrated stability or improvement in kidney function while being treated with agalsidase alfa. Of importance was the observation that only 1 of 20 patients with Stage 2 CKD and none of the three patients with Stage $3 \mathrm{CKD}$ demonstrated loss of GFR in excess $5 \mathrm{~mL} /$ minute $/ 1.73 \mathrm{~m}^{2}$ per year while treated with agalsidase alfa during this study.

Seven women were treated with angiotensin-converting enzyme (ACE) inhibitors or angiotensin-receptor blockers (ARBs) during the entire study, and six additional women began therapy with these agents after starting treatment with agalsidase alfa. The seven women who were on ACE inhibitor or ARB therapy during the entire study experienced a change in eGFR from $74.5 \pm 10.4$ to $65.0 \pm 13.5 \mathrm{~mL} / \mathrm{minute} / 1.73 \mathrm{~m}^{2}$ and a change in proteinuria from $1349 \pm 1760$ to $425 \pm 531 \mathrm{mg} / 24$ hours over the 4-year observation period. The six women who initiated ACE inhibitor or ARB therapy during the study demonstrated stable eGFR thereafter $\left(87.7 \pm 29.6 \mathrm{~mL} /\right.$ minute $/ 1.73 \mathrm{~m}^{2}$ at the last measurement before starting antiangiotensin therapy and $83.0 \pm 33.4 \mathrm{~mL} /$ minute $/ 1.73 \mathrm{~m}^{2}$ at study end).

\section{Proteinuria}

Three women were treated with corticosteroids for immunoglobulin A or other nephropathy during the study, and their data were not included in the longitudinal analysis. In the remaining 33 patients, mean urinary protein excretion was $377 \pm 546$ $\mathrm{mg} / 24$ hours at baseline (median $=180 \mathrm{mg} / 24$ hours) and fell to $263 \pm 167 \mathrm{mg} / 24$ hours after 4 years $(P=\mathrm{NS})$. In the subgroup of 11 patients with proteinuria in excess of 300 $\mathrm{mg} /$ day, mean protein excretion declined significantly during treatment, from a baseline value of $858 \pm 751 \mathrm{mg} / 24$ hours to $339 \pm 230 \mathrm{mg} / 24$ hours after 4 years of treatment $(P<0.01$, Fig. 4).

\section{Cardiomyopathy}

LVH (defined as LVM $\geq 48 \mathrm{~g} / \mathrm{m}^{2.7}$ ) was common and found at baseline in 25 of the 36 patients $(69 \%)$ who went on to complete 4 years of treatment. In this subgroup, mean LVM at baseline was $89.4 \pm 42.1 \mathrm{~g} / \mathrm{m}^{2.7}$ (range: $52.8-201.1 \mathrm{~g} / \mathrm{m}^{2.7}$ ). Because of the wide range of observed LVM in the cohort with $\mathrm{LVH}$, this subgroup was subdivided into tertiles for analysis (baseline LVM $>48-60 \mathrm{~g} / \mathrm{m}^{2.7},>60-85 \mathrm{~g} / \mathrm{m}^{2.7},>85 \mathrm{~g} / \mathrm{m}^{2.7}$ ). As shown in Table 4, each of these tertiles demonstrated a significant reduction in LVM after 12 months of agalsidase alfa. Thirteen of these 25 patients $(52 \%)$ demonstrated decreases of LVM in excess of $20 \%$, seven other patients $(28 \%)$ demonstrated decreases in LVM of between 10 and $20 \%$, and two patients experienced decreases of 7.2 and $3.5 \%$. Three patients $(12 \%)$ with baseline LVH exhibited small increases in LVM during the study ranging from 0.5 to $2.6 \%$. At study completion, 7 of these 25 patients had LVM that was classified as normal. Only 1 of 11 patients $(9 \%)$ who was classified as having normal LVM $\left(47.7 \mathrm{~g} / \mathrm{m}^{2.7}\right)$ at baseline was reclassified as having $\mathrm{LVH}$ after 4 years of treatment $\left(50.3 \mathrm{~g} / \mathrm{m}^{2.7}\right)$.

More than one third (13 of $36,36 \%$ ) of the patients who completed 4 years of treatment were classified with NYHA Class III heart failure (defined as "marked limitation of physical activity; comfortable at rest, but less than ordinary activity causes fatigue, palpitation, or dyspnea"47) at baseline. As shown in Table 5, the average NYHA score in this subgroup significantly improved after 12 months of agalsidase alfa treatment and remained improved throughout the study. After 4 years of agalsidase alfa treatment, no patients progressed to a more severe stage of heart failure. Of the 13 patients who were in NYHA Class III heart failure at baseline, nine had improved to Class II and three had improved to Class I after 4 years of treatment. All four patients in NYHA Class II heart failure had improved to Class I after 4 years.

\section{Pain}

In the 36 patients who completed 4 years of agalsidase alfa treatment, the BPI "pain at its worst" score at baseline was $4.6 \pm 2.9$ and declined to $3.3 \pm 2.9$ after 12 months $(P=$ $0.001)$. No further improvement was observed. At baseline, 22 $(61 \%)$ of these patients had a BPI "pain at its worst" score $\geq 5$, which is considered to be a threshold for interference with activities of daily living (e.g., physical activity, mood, social activity, relations with others, sleep ${ }^{48}$ ). After 48 months, 10 $(45 \%)$ of these patients had scores below this threshold. Of the $14(39 \%)$ patients with baseline BPI "pain at its worst" scores $<5$, only two $(14 \%)$ were above this threshold after 4 years of agalsidase alfa treatment. Similarly, 18 of $30(60 \%)$ of patients with a BPI "pain at its worst" score $\geq 1$ at baseline improved by at least 1 point after 4 years of treatment.

\section{Biochemical measurements}

\section{Plasma Gb3}

At baseline, 26 of 36 (72\%) women who completed 4 years of treatment had plasma Gb3 levels that were above the ULN, with three $(8 \%)$ patients having levels $>2$ times the ULN. Their average plasma Gb3 score was $1.81 \pm 0.58$. After 2 years of agalsidase alfa treatment, the average score had decreased to $1.53 \pm 0.51(P=0.005)$. By 4 years, the average score had dropped to $1.31 \pm 0.47(P<0.001)$, and only 11 patients had plasma Gb3 scores that remained above the ULN, and none had levels $>2$ times the ULN. 

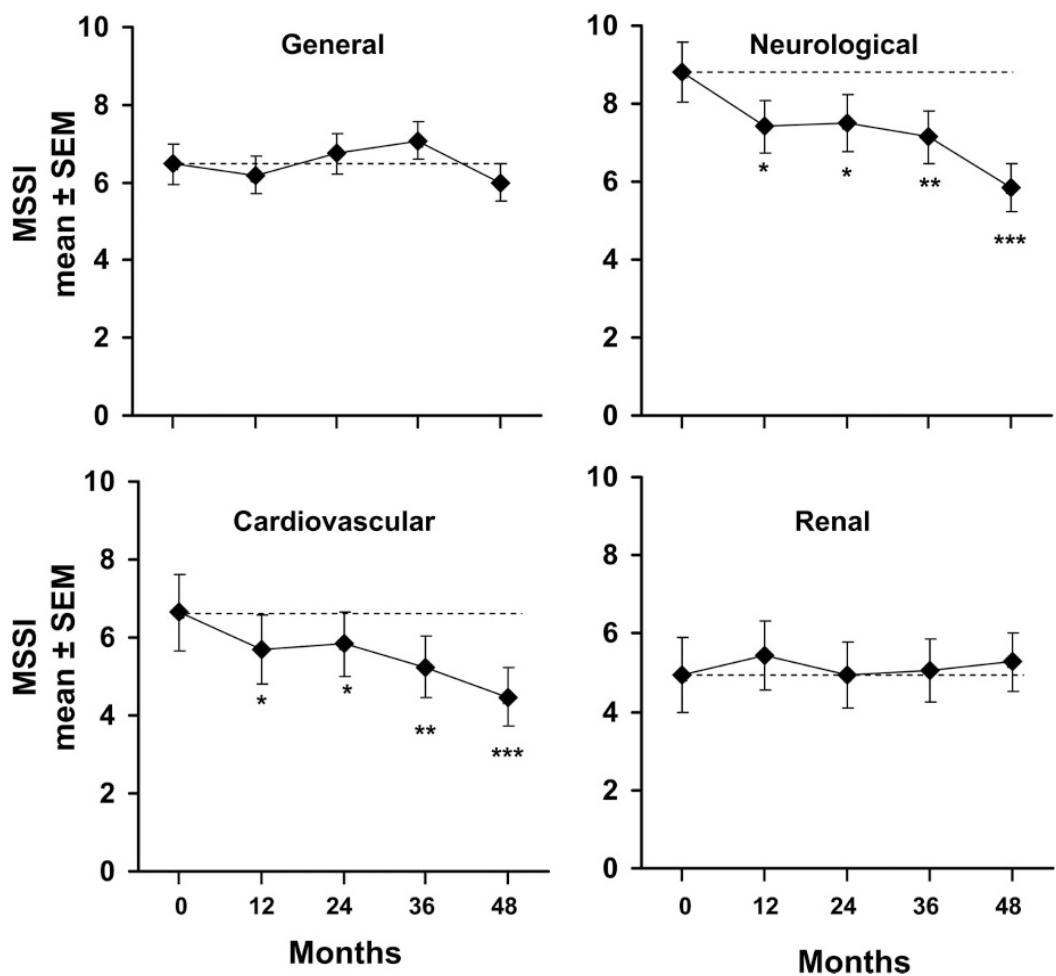

Fig. 3. The effect of agalsidase alfa on MSSI individual component scores. The dotted lines represent the baseline values. ${ }^{*} P<0.05,{ }^{* *} P<0.01,{ }^{* * *} P<0.001$ compared with baseline (Month 0 ). $N=36$.

\section{Urine Gb3}

The average baseline urine Gb3 score was $1.67 \pm 0.72$ with $20(56 \%)$ patients exhibiting levels higher than the ULN with 3 $(8 \%)$ of them $>2$ times the ULN. After 1 year of agalsidase alfa treatment, the average urine Gb3 score was significantly decreased to $1.29 \pm 0.52(P<0.001)$, and only 10 patients remained above the ULN. Urine Gb3 scores continued to drop throughout the study, and, after 4 years, only one patient remained with a score above the ULN.

\section{Safety}

Agalsidase alfa was well tolerated during this study. One woman experienced an infusion reaction characterized by chills and fever. No antiagalsidase alfa antibodies were detected at any time during this treatment period. Five women experienced a stroke during the study. One additional woman experienced a stroke within 1 year of discontinuing treatment. Three of these women had a history of stroke before initiating agalsidase alfa.

\section{DISCUSSION}

This study represents the largest and longest examination of the effects of ERT in women with Fabry disease. The cross-sectional analyses of the baseline data (Fig. 1) suggest that much of the organ involvement is progressive in women, just as it is in men. The total burden of the disease, as measured by the MSSI, increased with age, as did the LVM. Renal function assessed by eGFR showed a progressive deterioration with age. In contrast, the severity of neuropathic pain did not change with advancing age. Overall, the results revealed clinical benefit during ERT with agalsidase alfa in women with Fabry disease, as shown by the decrease in MSSI score, the stability in kidney function, the reduc-

Table 2 eGFR during treatment with agalsidase alfa in women with Fabry disease

\begin{tabular}{lcccccc}
\hline & & \multicolumn{4}{c}{ Months of treatment } \\
\cline { 3 - 6 } eGFR & $n$ & Baseline & 12 & 24 & 36 & 48 \\
\hline$>135$ & 4 & $159.0 \pm 19.7$ & $140.3 \pm 26.8$ & $135.3 \pm 18.3^{a}$ & $133.0 \pm 17.0^{a}$ & $128.3 \pm 4.6^{b}$ \\
$90-135$ & 9 & $106.6 \pm 14.9$ & $98.7 \pm 15.6$ & $98.5 \pm 10.9$ & $106.7 \pm 15.5$ & $100.4 \pm 16.4$ \\
$60-89$ & 20 & $76.6 \pm 8.5$ & $85.5 \pm 13.2^{b}$ & $88.8 \pm 14.5^{c}$ & $83.0 \pm 17.9^{a}$ & $85.9 \pm 20.2^{b}$ \\
$30-59$ & 3 & $50.2 \pm 7.3$ & $50.4 \pm 10.8$ & $55.8 \pm 8.1$ & $49.7 \pm 11.5$ & $47.3 \pm 14.0$ \\
\hline${ }^{a} P<0.05,{ }^{b} P<0.01,{ }^{c} P<0.001$ compared with baseline.
\end{tabular}


Table 3 Responder analysis of the effect of agalsidase alfa on kidney function in female Fabry disease patients

\begin{tabular}{lrcc}
\hline & & \multicolumn{2}{c}{ Responder definition } \\
\cline { 3 - 4 } Baseline category & $N$ & I (\%) & II (\%) \\
\hline $90-135 \mathrm{~mL} / \mathrm{min} / 1.73 \mathrm{~m}^{2}$ & 9 & 89 & 89 \\
$60-89 \mathrm{~mL} / \mathrm{min} / 1.73 \mathrm{~m}^{2}$ & 20 & 95 & 95 \\
$30-59 \mathrm{~mL} / \mathrm{min} / 1.73 \mathrm{~m}^{2}$ & 3 & 67 & 100 \\
\hline
\end{tabular}

Responder definitions: I, less than $20 \%$ decrease in GFR from baseline; II, no shift to more severe CKD stage. The categories are based on the classification of chronic kidney disease. ${ }^{42}$ Women who had eGFR $>135 \mathrm{~mL} / \mathrm{min} / 1.73 \mathrm{~m}^{2}$ at baseline were defined as exhibiting hyperfiltration ${ }^{46}$ and excluded from this analysis because large decreases in eGFR could be interpreted as either improvement in function or as continued progression of disease.

tion in LVM in patients with baseline LVH, and the improvement in NYHA heart failure classification.

The MSSI is an instrument that assigns a numeric value to signs and symptoms of Fabry disease in four organ systems: renal, cardiovascular, neurologic, and general. The mean MSSI score at baseline (26.2) is indicative of moderate severity of the signs and symptoms of Fabry disease. ${ }^{31}$ Treatment with agalsidase alfa resulted in a continuous improvement in the MSSI score (Fig. 2), a response primarily because of an improvement in the neurologic and cardiovascular subscales (Fig. 3). Similar improvements in MSSI have been previously reported for both men and women treated with agalsidase alfa. ${ }^{31}$

Kidney dysfunction in female heterozygotes is thought to be less severe than in male hemizygotes, and it seems that fewer women than men progress to ESRD. In several cross-sectional studies, the percentage of women with ESRD is reported to be about one third that of men. ${ }^{21,25}$ The difference is even greater in the Fabry Outcome Survey, where ESRD was reported in $17 \%$ of men and only $1 \%$ of women with Fabry disease. ${ }^{27}$ Similar findings have emerged from the Fabry Registry, where ESRD was reported in $13-17 \%$ of men and only $2.2-4.4 \%$ of women. ${ }^{15,49}$ A recent single-center study of 44 women found ESRD in $12.5 \% .{ }^{18}$ No well-controlled longitudinal studies of

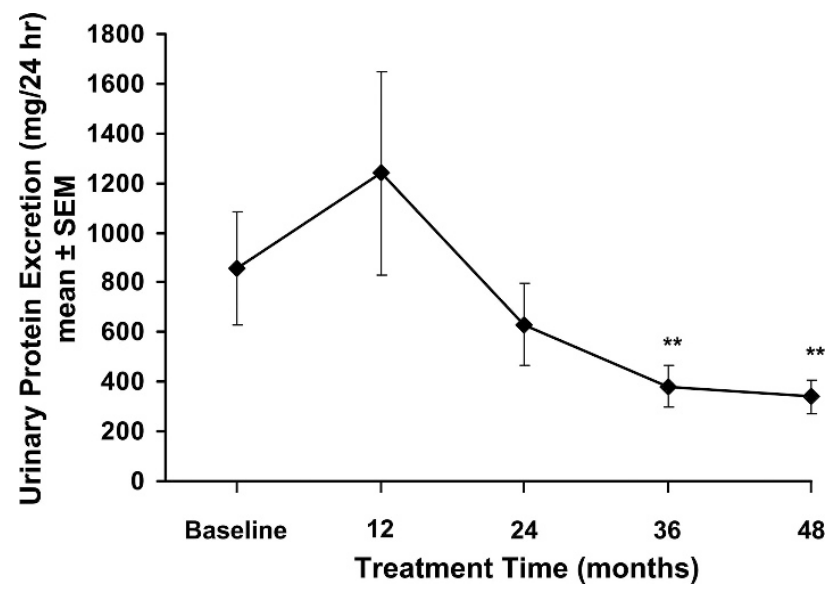

Fig. 4. Effect of agalsidase alfa on proteinuria in women with baseline protein excretion exceeding $300 \mathrm{mg} /$ day. $* * P<0.01$ compared with baseline based on ANOVA of log-transformed values. $N=11$. the rate of loss of GFR in untreated female patients with Fabry disease have been reported, but the cross-sectional data in Figure 1 and from the Fabry Registry ${ }^{49}$ clearly show a loss of GFR with advancing age. Other evidence of kidney dysfunction is commonly found in female patients with Fabry disease. For example, in studies in which renal biopsies have been taken, almost all women have histological evidence of kidney involvement. ${ }^{50,51}$ Proteinuria, another indicator of kidney dysfunction, has been reported in $41 \%$ of women enrolled in Fabry Outcome Survey, compared with $54 \%$ of men, ${ }^{52}$ and has been reported in adolescent girls. ${ }^{4,23}$ In the present study, proteinuria (protein excretion $>300 \mathrm{mg} / 24$ hours) was present in $35 \%$ of the women at baseline.

Few studies have reported the effect of ERT on kidney function in women with Fabry disease. Baehner et al. ${ }^{39}$ found that mean creatinine clearance remained constant through 41 weeks of treatment with agalsidase alfa. Fourteen women were included in a study reported by Schwarting et al., ${ }^{53}$ and although they did not specifically report the effects in women, their individual data were reported. In that cohort of female patients with Fabry disease, eGFR declined by $7.1 \pm 2.3 \mathrm{~mL} /$ minute/ $1.73 \mathrm{~m}^{2}$ in the year before initiating treatment with agalsidase alfa. During 1 year of agalsidase alfa treatment, the rate of decline was reduced to $1.1 \pm 1.3 \mathrm{~mL} /$ minute $/ 1.73 \mathrm{~m}^{2}(P=$ $0.054, t$ test), a rate of loss similar to that reported for the general population over 30 years of age. ${ }^{41}$

Hyperfiltration is reported in both adult men ${ }^{37}$ and children ${ }^{9}$ with Fabry disease and may be the initial sign of kidney dysfunction in affected patients. In these patients, a fall in eGFR toward the normal range may indicate an improvement in kidney function, although it cannot be distinguished from continued progression of kidney disease. In the present study, the four women who were considered to be "hyperfiltrators" at baseline all demonstrated a reduction in eGFR toward normal during the 4-year study.

The present study is the only long-term evaluation of ERT on kidney function in women with Fabry disease that has been reported to date. Agalsidase alfa seemed to stabilize or improve eGFR in this cohort over 4 years of treatment (Tables 2 and 3) and to reduce proteinuria in those patients with baseline protein excretion exceeding $300 \mathrm{mg} / 24$ hours (Fig. 4). As illustrated in Table 3, 90\% of patients who were not hyperfiltrators at baseline demonstrated stable or improved kidney function during the treatment period, an observation that can be interpreted as a positive response to treatment.

The improvement in eGFR and the reduction in proteinuria seen in subsets of women in this study have not been reported in other large studies of ERT that included primarily male patient populations. ${ }^{37,54,55}$ In those studies, GFR in patients with Stage 1 or Stage 2 CKD remained stable during ERT, and no effect on proteinuria was found. Although it is attractive to hypothesize that the renal involvement in some women in this study had not reached the "point of no return," and therefore agalsidase alfa was able to reverse ongoing pathologic changes, no evidence exists to support that claim.

ACE inhibitors or ARBs are now considered to be standard therapy in patients with Fabry disease. ${ }^{56}$ A preliminary study in which the dose of ACE inhibitors or ARBs was titrated to establish a reduction in proteinuria showed that ERT could stabilize kidney function in patients with Fabry disease despite baseline proteinuria. ${ }^{56}$ The present study was started before the renoprotective effects of these drug classes was well established, and the study was not designed to evaluate the effect of these drugs on kidney function in a controlled setting. Therefore, no conclusions about their role 
Table 4 The effect of agalsidase alfa on left-ventricular mass in patients with and without baseline left-ventricular hypertrophy

\begin{tabular}{|c|c|c|c|c|c|c|}
\hline \multirow[b]{2}{*}{ LVM classification } & \multirow[b]{2}{*}{$N$} & \multicolumn{5}{|c|}{ Months of treatment } \\
\hline & & Baseline & 12 & 24 & 36 & 48 \\
\hline$>85 \mathrm{~g} / \mathrm{m}^{2.7}$ & 9 & $131.9 \pm 43.3$ & $98.1 \pm 31.1^{a}$ & $98.1 \pm 31.7^{a}$ & $96.3 \pm 34.0^{a}$ & $91.9 \pm 29.7^{a}$ \\
\hline$>60-85 \mathrm{~g} / \mathrm{m}^{2.7}$ & 9 & $76.3 \pm 9.9$ & $57.4 \pm 13.3^{a}$ & $56.5 \pm 14.6^{a}$ & $58.2 \pm 13.2^{a}$ & $62.0 \pm 17.7^{a}$ \\
\hline$>48-60 \mathrm{~g} / \mathrm{m}^{2.7}$ & 8 & $56.0 \pm 2.1$ & $46.3 \pm 4.7^{a}$ & $44.0 \pm 5.2^{a}$ & $47.0 \pm 2.8^{a}$ & $48.7 \pm 4.1^{a}$ \\
\hline$<48 \mathrm{~g} / \mathrm{m}^{2.7}$ & 11 & $40.7 \pm 6.4$ & $36.2 \pm 5.8^{b}$ & $35.3 \pm 7.8^{b}$ & $37.0 \pm 10.1^{c}$ & $36.2 \pm 7.1^{b}$ \\
\hline
\end{tabular}

in affecting the progression of kidney disease in women with Fabry disease can be made from the present results. A larger clinical trial designed to assess the role of ACE inhibitors and ARBs in the preservation of kidney function during ERT of Fabry disease is ongoing.

Cardiovascular involvement is an important contributor to the morbidity and mortality associated with Fabry disease in both men and women. ${ }^{26,57}$ In the present study, LVM was significantly reduced in patients with baseline LVH after 1 year of agalsidase alfa, and remained reduced through 4 years of treatment (Table 4). Importantly, LVM was normalized in 7 of 25 women who were characterized with $\mathrm{LVH}$ at baseline during the 4 years of agalsidase alfa treatment. In addition, agalsidase alfa resulted in clinical improvement of symptoms of heart failure (Table 5). At baseline, more than one third of the women were classified as having NYHA Class III heart failure. At this stage of heart failure, almost any activity results in fatigue or dyspnea. Although the progressive reduction in the percentage of patients in NYHA Class III, combined with the progressive increase in the percentage of patients with minimal symptoms (NYHA Class I), may be interpreted as evidence that agalsidase alfa improved cardiac function in women, it is important to realize that functional improvement may reflect noncardiovascular factors, including reduction in neuropathic pain and improvement in sweating.

Neurologic pain adversely affects quality of life in both men $^{33}$ and women ${ }^{18}$ with Fabry disease. In the present study, the average BPI "pain at its worst" score of $4.6 \pm 2.9$ at baseline is somewhat higher than reported for a cohort of 19 women enrolled in the Fabry Registry (mean score $=3.7 \pm 3.5$ ). ${ }^{18}$ Agalsidase alfa has been reported to reduce neuropathic pain in men, ${ }^{36}$ and, in the present study in women, the BPI "pain at its worst" score was significantly improved during 4 years of treatment. Importantly, nearly $50 \%$ of women who had baseline BPI "pain at its worst" scores of $\geq 5$, had scores below this threshold after 4 years. This threshold value is important, because at levels of $\geq 5$, the pain interferes at least moderately with most activities of daily living. ${ }^{58}$ Despite this apparent benefit, it must be understood that the use of pain medications was not controlled during this study, and some of the benefit might reflect changes in their use by the study participants. In addition, it is impossible to eliminate the possibility of a placebo effect on pain during this study.

\section{Study limitations}

This study was an open-label, observational, clinical trial conducted in a single center. This site is a referral center, and therefore, the patient population may not be representative of the general female Fabry disease population. The absence of a concurrently followed untreated control group limits the strength of the conclusions regarding the effect of agalsidase alfa on organ-system involvement in women with Fabry disease. Finally, the changing use of ACE inhibitors or ARBs as well as the unknown changes in the use of statins and antiplatelet agents may have contributed to some of the benefits noted, particularly the apparent reductions in proteinuria and maintenance of stable GFR.

\section{CONCLUSIONS}

This study represents the largest and longest follow-up of ERT in women with Fabry disease. Female patients can experience all of the signs and symptoms of Fabry disease as do men and, as is true in men, the burden of the disease increases with age. ERT with agalsidase alfa significantly decreased the burden of disease as measured by MSSI, primarily by reducing the neurologic involvement (e.g., reduction in pain) and by improving cardiac structure and function. In addition, kidney function was improved in patients with moderately reduced baseline eGFR or proteinuria and stabilized in patients with mild or severe CKD. Based on these observations, women with any signs and symptoms of Fabry disease should be considered for ERT.

\section{ACKNOWLEDGMENTS}

Editorial assistance was provided by Edward Weselcouch, $\mathrm{PhD}$, of PharmaWrite and was paid for by Shire HGT.

Table 5 The effect of agalsidase alfa on heart failure in women with Fabry disease

\begin{tabular}{lllllll}
\hline & & \multicolumn{4}{c}{ Months of treatment } \\
\cline { 3 - 7 } & $N$ & Baseline & 12 & 24 & 36 & 48 \\
\hline NYHA classification (mean $\pm \mathrm{SD})$ & 36 & $1.83 \pm 0.94$ & $1.53 \pm 0.70^{a}$ & $1.28 \pm 0.51^{a}$ & $1.31 \pm 0.52^{a}$ & $1.31 \pm 0.52^{a}$ \\
\hline${ }^{a} P<0.001$ compared with baseline. & & & & & & \\
\hline
\end{tabular}




\section{REFERENCES}

1. Brady RO, Gal AE, Bradley RM, Martensson E, Warshaw AL, Laster L. Enzymatic defect in Fabry's disease: ceramidetrihexosidase deficiency. N Engl J Med 1967;276:1163-1167.

2. MacDermot J, MacDermot KD. Neuropathic pain in Anderson-Fabry disease: pathology and therapeutic options. Eur J Pharmacol 2001;429:121-125.

3. Ries M, Gupta S, Moore DF, et al. Pediatric Fabry disease. Pediatrics 2005; $115: \mathrm{e} 344-\mathrm{e} 355$

4. Ries M, Ramaswami U, Parini R, et al. The early clinical phenotype of Fabry disease: a study on 35 European children and adolescents. Eur $J$ Pediatr 2003;162:767-772.

5. Kampmann C, Wiethoff CM, Perrot A, Beck M, Dietz R, Osterziel KJ. The heart in Anderson Fabry disease. Z Kardiol 2002;91:786-795.

6. Fellgiebel A. Stroke and brain structural alterations in Fabry disease. Clin Ther 2007;29(suppl A):S9-S10

7. Rolfs A, Bottcher T, Zschiesche M, et al. Prevalence of Fabry disease in patients with cryptogenic stroke: a prospective study. Lancet 2005;366:17941796

8. Cable WJ, Kolodny EH, Adams RD. Fabry disease: impaired autonomic function. Neurology 1982;32:498-502.

9. Ries M, Clarke JT, Whybra C, et al. Enzyme-replacement therapy with agalsidase alfa in children with Fabry disease. Pediatrics 2006;118:924-932.

10. Kampmann C, Wiethoff CM, Whybra C, Baehner FA, Mengel E, Beck M. Cardiac manifestations of Anderson-Fabry disease in children and adolescents. Acta Paediatr 2008;97:463-469.

11. Branton MH, Schiffmann R, Sabnis SG, et al. Natural history of Fabry renal disease: influence of $\alpha$-galactosidase A activity and genetic mutations on clinical course. Medicine 2002;81:122-138.

12. Meikle PJ, Hopwood JJ, Clague AE, Carey WF. Prevalence of lysosomal storage disorders. JAMA 1999;281:249-254.

13. Spada M, Pagliardini S, Yasuda M, et al. High incidence of later-onset Fabry disease revealed by newborn screening. Am J Hum Genet 2006;79:31-40.

14. Dobyns WB. The pattern of inheritance of X-linked traits is not dominant or recessive, just X-linked. Acta Paediatr Suppl 2006;95:11-15.

15. Wilcox WR, Oliveira JP, Hopkin RJ, et al. Females with Fabry disease frequently have major organ involvement: lessons from the Fabry Registry. Mol Genet Metab 2008;93:112-128.

16. Whybra C, Kampmann C, Willers I, et al. Anderson-Fabry disease: clinical manifestations of disease in female heterozygotes. J Inherit Metab Dis 2001; 24:715-724

17. Wendrich K, Whybra C, Ries M, Gal A, Beck M. Neurological manifestation of Fabry disease in females. Contrib Nephrol 2001;136:241-244.

18. Wang RY, Lelis A, Mirocha J, Wilcox WR. Heterozygous Fabry women are not just carriers, but have a significant burden of disease and impaired quality of life. Genet Med 2007;9:34-45.

19. MacDermot KD, Holmes A, Miners AH. Anderson-Fabry disease: clinical manifestations and impact of disease in a cohort of 60 obligate carrier females. J Med Genet 2001:38:769-775.

20. Deegan PB, Baehner AF, Barba Romero MA, et al. Natural history of Fabry disease in females in the Fabry Outcome Survey. J Med Genet 2006;43:347-352.

21. Kobayashi M, Ohashi T, Sakuma M, Ida H, Eto Y. Clinical manifestations and natural history of Japanese heterozygous females with Fabry disease. $J$ Inherit Metab Dis January 21, 2008 [epub Ahead of Print].

22. Guffon N. Clinical presentation in female patients with Fabry disease. J Med Genet 2003;40:e38

23. Ramaswami U, Whybra C, Parini R, et al. Clinical manifestations of Fabry disease in children: data from the Fabry Outcome Survey. Acta Paediatr 2006;95:86-92.

24. Hopkin RJ, Bissler J, Banikazemi M, et al. Characterization of Fabry Disease in 352 Pediatric Patients in the Fabry Registry. Pediatr Res 2008;64:550-555.

25. MacDermot KD, Holmes A, Miners AH. Anderson-Fabry disease: clinical manifestations and impact of disease in a cohort of 98 hemizygous males. J Med Genet 2001;38:750-760.

26. Kampmann C, Linhart A, Baehner F, et al. Onset and progession of the Anderson-Fabry disease related cardiomyopathy. Int J Cardiol 2008;130: 367-373.

27. Mehta A, Ricci R, Widmer U, et al. Fabry disease defined: baseline clinical manifestations of 366 patients in the Fabry Outcome Survey. Eur J Clin Invest 2004; $34: 236-242$

28. Vedder AC, Linthorst GE, van Breemen MJ, et al. The Dutch Fabry cohort: diversity of clinical manifestations and $\mathrm{Gb}_{3}$ levels. $J$ Inherit Metab Dis 2007;30:68-78

29. Eng CM, Fletcher J, Wilcox WR, et al. Fabry disease: baseline medical characteristics of a cohort of 1765 males and females in the Fabry Registry. J Inherit Metab Dis 2007;30:184-192.

30. Beck M. Demographics of FOS: the Fabry outcomes survey. In: Mehta A, Beck M, Sunder-Plassmann G, editors. Fabry disease: perspectives from 5 years of FOS. Oxford, UK: Oxford PharmaGenesis Ltd., 2006:155-161.
31. Whybra C, Kampmann C, Krummenauer F, et al. The Mainz Severity Score Index: a new instrument for quantifying the Anderson-Fabry disease phenotype, and the response of patients to enzyme replacement therapy. Clin Genet 2004:65:299-307.

32. Street NJ, Yi MS, Bailey LA, Hopkin RJ. Comparison of health-related quality of life between heterozygous women with Fabry disease, a healthy control population, and patients with other chronic disease. Genet Med 2006;8:346-353.

33. Gold KF, Pastores GM, Botteman MF, et al. Quality of life of patients with Fabry disease. Oual Life Res 2002;11:317-327.

34. Miners AH, Holmes A, Sherr L, Jenkinson C, MacDermot KD. Assessment of health-related quality-of-life in males with Anderson Fabry disease before therapeutic intervention. Oual Life Res 2002;11:127-133.

35. Schiffmann R, Murray GJ, Treco D, et al. Infusion of $\alpha$-galactosidase A reduces tissue globotriaosylceramide storage in patients with Fabry disease. Proc Natl Acad Sci USA 2000;97:365-370.

36. Schiffmann R, Kopp JB, Austin HAI, et al. Enzyme replacement therapy in Fabry disease: a randomized controlled trial. JAMA 2001;285:2743-2749.

37. Schiffmann R, Ries M, Timmons M, Flaherty JT, Brady RO. Long-term therapy with agalsidase alfa for Fabry disease: safety and effects on renal function in a home infusion setting. Nephrol Dial Transplant 2006;21:345-354.

38. Hughes DA, Elliott PM, Shah J, et al. Effects of enzyme replacement therapy on the cardiomyopathy of Anderson-Fabry disease: a randomized, double-blind, placebo-controlled clinical trial of agalsidase-alfa. Heart 2008;94:153-158.

39. Baehner F, Kampmann C, Whybra C, Miebach E, Wiethoff CM, Beck M. Enzyme replacement therapy in heterozygous females with Fabry disease: results of a phase IIIB study. J Inherit Metab Dis 2003;26:617-627.

40. Cleeland CS. Pain assessment: the advantages of using pain scales in lysosomal storage diseases. Acta Paediatr 2002;91:43-47.

41. Levey AS. Clinical practice. Nondiabetic kidney disease. $N$ Engl $\mathrm{J}$ Med 2002;347:1505-1511.

42. National Kidney Foundation (NKF) Kidney Disease Outcome Quality Initiative (K/DOQI) Advisory Board. K/DOQI clinical practice guidelines for chronic kidney disease: evaluation, classification, and stratification. Kidney Disease Outcome Quality Initiative. Am J Kidney Dis 2002;39:S1-S266.

43. Schiffmann R, Askari H, Timmons M, et al. Weekly enzyme replacement therapy may slow decline of renal function in patients with Fabry disease who are on long-term biweekly dosing. J Am Soc Nephrol 2007;18:1576-1583.

44. Devereux RB. Detection of left ventricular hypertrophy by M-mode echocardiography. Anatomic validation, standardization, and comparison to other methods. Hypertension 1987;9:II19-II26.

45. Devereux RB, Koren MJ, de Simone G, Roman MJ, Laragh JH. Left ventricular mass as a measure of preclinical hypertensive disease. Am J Hypertens 1992; $5: 175 \mathrm{~S}-181 \mathrm{~S}$

46. Cotroneo P, Manto A, Todaro L, et al. Hyperfiltration in patients with type I diabetes mellitus: a prevalence study. Clin Nephrol 1998:50:214-217.

47. Tenenbaum A, Motro M, Fisman EZ, et al. Functional class in patients with heart failure is associated with the development of diabetes. $\mathrm{Am} \mathrm{J} \mathrm{Med}$ 2003; 114:271-275.

48. Cleeland CS, Ryan KM. Pain assessment: global use of the Brief Pain Inventory. Ann Acad Med Singapore 1994;23:129-138.

49. Ortiz A, Oliveira JP, Waldek S, Warnock DG, Cianciaruso B, Wanner C. Nephropathy in males and females with Fabry disease: cross-sectional description of patients before treatment with enzyme replacement therapy. Nephrol Dialysis Transplant 2008;23:1600-1607.

50. Gubler MC, Lenoir G, Grunfeld JP, Ulmann A, Droz D, Habib R. Early renal changes in hemizygous and heterozygous patients with Fabry's disease. Kidney Int 1978;13:223-235.

51. Tosoni A, Nebuloni M, Zerbi P, Vago L, Comotti C, Sessa A. Ultrastructural study of renal involvement in two females with Anderson-Fabry disease. Ultrastruct Pathol 2005;29:203-207.

52. Beck M, Ricci R, Widmer U, et al. Fabry disease: overall effects of agalsidase alfa treatment. Eur J Clin Invest 2004;34:838-844.

53. Schwarting A, Dehout F, Feriozzi S, et al. Enzyme replacement therapy and renal function in 201 patients with Fabry disease. Clin Nephrol 2006;66:77-84

54. Banikazemi M, Bultas J, Waldek S, et al. Agalsidase-beta therapy for advanced Fabry disease: a randomized trial. Ann Intern Med 2007;146:77-86.

55. Germain DP, Waldek S, Banikazemi M, et al. Sustained, long-term renal stabilization after 54 months of agalsidase beta therapy in patients with Fabry disease. J Am Soc Nephrol 2007;18:1547-1557.

56. Tahir H, Jackson LL, Warnock DG. Antiproteinuric therapy and Fabry nephropathy: sustained reduction of proteinuria in patients receiving enzyme replacement herapy with agalsidase-beta. J Am Soc Nephrol 2007;18:2609-2617.

57. Kampmann C, Baehner F, Whybra C, et al. Cardiac manifestations of Anderson-Fabry disease in heterozygous females. J Am Coll Cardiol 2002;40: $668-1674$

58. Cleeland CS. The brief pain inventory, a measure of cancer pain and its impact. Qual Life Newslett 1994;9:5-6. 